\title{
Mobile sensor network noise reduction and recalibration using a Bayesian network
}

\author{
Y. Xiang, Y. Tang, and W. Zhu \\ College of Information Engineering, Zhejiang University of Technology, Hangzhou, China
}

Correspondence to: Y. Xiang (xiangyun@zjut.edu.cn)

Received: 13 May 2015 - Published in Atmos. Meas. Tech. Discuss.: 31 August 2015

Revised: 17 January 2016 - Accepted: 23 January 2016 - Published: 4 February 2016

\begin{abstract}
People are becoming increasingly interested in mobile air quality sensor network applications. By eliminating the inaccuracies caused by spatial and temporal heterogeneity of pollutant distributions, this method shows great potential for atmospheric research. However, systems based on low-cost air quality sensors often suffer from sensor noise and drift. For the sensing systems to operate stably and reliably in real-world applications, those problems must be addressed. In this work, we exploit the correlation of different types of sensors caused by cross sensitivity to help identify and correct the outlier readings. By employing a Bayesian network based system, we are able to recover the erroneous readings and recalibrate the drifted sensors simultaneously. Our method improves upon the state-of-art Bayesian belief network techniques by incorporating the virtual evidence and adjusting the sensor calibration functions recursively.

Specifically, we have (1) designed a system based on the Bayesian belief network to detect and recover the abnormal readings, (2) developed methods to update the sensor calibration functions infield without requirement of ground truth, and (3) extended the Bayesian network with virtual evidence for infield sensor recalibration. To validate our technique, we have tested our technique with metal oxide sensors measuring $\mathrm{NO}_{2}, \mathrm{CO}$, and $\mathrm{O}_{3}$ in a real-world deployment. Compared with the existing Bayesian belief network techniques, results based on our experiment setup demonstrate that our system can reduce error by $34.1 \%$ and recover 4 times more data on average.
\end{abstract}

\section{Introduction}

Traditional atmospheric research usually relies upon stationary monitoring instruments to monitor the environmental pollutants. The distributions of air pollutants are non-uniform and highly dynamic both spatially and temporally. Unfortunately, official monitoring networks are usually very sparse due to their high costs. Therefore, mobile and distributed atmospheric air quality sensor networks are becoming increasingly popular and mainstream (Jiang et al., 2011; Willett et al., 2010; Piedrahita et al., 2014; Xiang, 2014). Those sensor networks are carried by users and are capable of measuring the immediate surrounding atmosphere. The metal oxide sensors used in the sensing devices are typically miniature, low power, and inexpensive in exchange for accuracy, sensitivity, and reliability. For those mobile sensors, the measured data usually contain significant noise from several sources. Subsequently, those noisy readings can trigger false alarms, lead to incorrect scientific conclusions, and generate suboptimal solutions (Zhang et al., 2010; Chandola et al., 2009).

Sensor noises are mainly caused by random factors and sensor drift. The metal oxide sensors are very sensitive to environmental parameters, e.g., temperature and humidity, which cannot be perfectly measured near the sensor surface. Moreover, there can be many unexpected problems in the real-world deployment, such as electrical components breakdown, power supplies surge, and signal noise in the circuits (Elnahrawy and Nath, 2003). Another significant source, observed and reported both in existing literature (Romain and Nicolas, 2010) and our own deployment, is sensor drift. Drift is a phenomenon caused by many factors that change the property of the sensing surface temporarily or permanently, including material degradation, exposure to sulfur 
compounds or acids, aging, or condensate on the sensor surface (Haugen et al., 2000; Arshak et al., 2004). Sensor drift changes the sensor function, which can cause significant deviation from the ground truth without proper error compensation. For example, in our own deployment, we find that the sensor drift can increase the average sensor error by orders of magnitude within several months. Drifted sensors must be recalibrated before they can be trusted and used again.

The metal oxide sensors, utilizing either the oxidation or reduction reactions with pollutant gases, can respond to and quantify the air pollutants with reasonable sensitivity and accuracy (Tans and Thoning, 2008). However, many pollutants share the same reaction property. For example, both $\mathrm{CO}$ and $\mathrm{NO}_{2}$ can cause oxidation reactions with the surface material. Thus, the sensors usually respond to a wide range of pollutants other than the targeting gas. This property is called cross sensitivity (Zampolli et al., 2004). Because of cross sensitivity, the readings of different types of sensors are usually correlated. This property can be used to identify the compositions of pollutants in the environment (Di Lecce and Calabrese, 2011).

We leverage the correlations of different metal oxide sensors to help identify and recover the abnormal readings. In many recent mobile sensing network designs, researchers have built sensing devices equipped with multiple types of sensors to detect various pollutants co-existing in the environment (Jiang et al., 2011; Willett et al., 2010). For such applications, it is possible to exploit the correlation of readings and recover noisy readings using Bayesian belief networks (Janakiram et al., 2006). The basic Bayesian network approach works well for the outliers caused by random noises but fails when sensors drift, which is common in realworld applications.

In this work, we aim to design a system that can efficiently detect and recover the noisy readings, recalibrate drifted sensors, and identify the gas compositions in the air simultaneously. This work makes the following contributions:

1. we have designed and implemented a Bayesian belief network based system to detect and recover outliers; and

2. we incorporate and address the sensor function calibration problem within the Bayesian network framework.

By analyzing the collected data, we have observed significant drift within a short period of time, e.g., a couple of months for most of the sensors. To validate our hypothesis and techniques, we have performed a field deployment. The deployment lasts about 3 months. During the deployment, we have mainly monitored and analyzed the following air quality related gases: $\mathrm{NO}_{2}, \mathrm{CO}$, and $\mathrm{O}_{3}$. The deployment results have confirmed our models about the sensor drift and the effectiveness of our techniques.

The rest of this paper is organized as follows. Section 3 discusses existing related work. Section 4 provides an overview of the system. Section 5 describes the Bayesian belief network approach and how to use it to detect and recover outliers. Section 6 discusses the limitations of existing Bayesian network approaches and presents our solution. Section 7 describes our real-world deployment and the evaluation results of different techniques.

\section{Motivation example}

This work is motivated by an atmosphere research project. Researchers have built several mobile atmosphere monitoring devices and deployed them in the field to monitor the atmosphere around the users. The devices can measure multiple pollutant gases using metal oxide sensors. Those sensors are precalibrated in the lab and are hence accurate before deployment. However, after a couple of months, it is discovered that the sensitivities of the sensors have shifted significantly, which can lead to erroneous scientific conclusions, false alarms, incorrect decisions, etc. Therefore, it is beneficial and important to develop a technique that can utilize the relationship between different types of sensors to reduce the sensor noise and recalibrate the sensors during deployment.

\section{Related work}

The related work can be placed in three categories: colocated sensor calibration, sensor abnormality detection, and Bayesian network based approaches.

\subsection{Co-located sensor calibration}

Xiang et al. $(2012,2013)$ developed a model to estimate sensor drift and designed a compensation technique to minimize the sensor drift assuming no access to ground truth readings. Bychkovskiy et al. (2003) have proposed a two-phase postdeployment sensor drift compensation technique in which co-located sensors are calibrated in pairs using linear functions. Miluzzo et al. (2008) have proposed CaliBree, an autocalibration algorithm for mobile sensor networks, in which mobile sensor nodes opportunistically interact with accurate stationary sensors and hence enable calibration to reduce sensor drift. Those techniques require that the co-located sensors are of the same type and thus should have the same response from the physical environment. In contrast to the previous work, our technique can work on mobile sensing devices containing various types of metal oxide sensors.

\subsection{Sensor abnormality detection}

Bettencourt et al. (2007) have presented an outlier detection technique to identify errors during event detection in ecological wireless sensor networks. Their technique uses the spatiotemporal correlations of sensor data to detect outliers. Rajasegarar et al. (2007) have proposed a technique based on a support vector machine (SVM) to detect sensor outliers. Their approach uses a one-class quarter-sphere SVM to 
classify and identify the local outliers. Unlike our technique, their method cannot estimate the actual ground truth readings and recover outliers. Papadimitriou et al. (2003) have developed a technique that uses multi-granularity deviation factor to dynamically detect the outlier readings based on the correlations of local nodes. Their technique cannot address the sensor drift problem, however, when one or more sensors' readings are shifted persistently. Kumar et al. (2013) proposed a technique that performs a two-stage drift correction. First, they use a Kriging based approach to provide estimated ground truth readings. Then a Kalman filter based technique is used to compensate for sensor drift. However, Kriging requires certain spatial density in sensor nodes deployment. Moreover, a Kalman filter based approach relies on the assumption of a state-space underlying model and knowledge of the model parameters, which is unrealistic in real-world applications when the environment of the deployment field is often unknown and very dynamic.

\subsection{Bayesian network based approaches}

Elnahrawy and Nath (2003) have used a naive Bayesian network to identify local outliers and detect faulty sensors. This technique uses a trained Bayesian classifier for probabilistic inference. Each node locally computes the probabilities of each of its incoming readings and determines the readings as outliers when their probabilities are not the highest among all the possible outcomes. Their approach can only work for the homogeneous sensors. Janakiram et al. (2006) have proposed a technique to detect sensor outliers based on Bayesian belief network. They leverage the conditional correlation of the readings from different types of sensors. However, their approach does not take into consideration sensor drift and sensor function recalibration, which are considered and addressed by our method.

\section{System flow}

Figure 1 shows the overview of our system. It describes the composition of the system. In the real-world applications, the gathered atmosphere data, e.g., $\mathrm{O}_{3}$, are processed by the system. The system can reduce the sensing error caused by drift as well as other atmospheric parameters and recalibrate the sensor function. The output of the system is the $\mathrm{O}_{3}$ data with significantly improved accuracy and a more sensitive sensor function.

The input sensor readings are first processed using a Bayesian belief network, which is trained using normal data from the infield deployment. The Bayesian network can generate the estimated ground truth values based on the conditional probability tables and readings from all the correlated sensors. The estimated ground truth readings are then used to recalibrate the sensors, i.e., generate the new sensor functions which can translate the input sensor analog readings into actual pollutant concentrations. The new sensor functions are

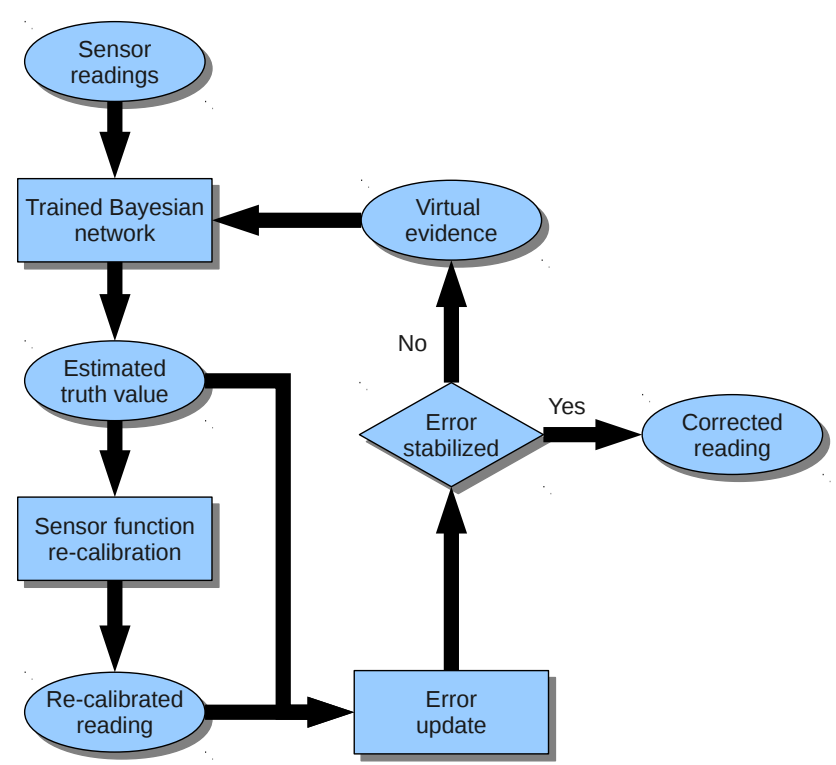

Figure 1. System flow.

used to generate the sensor readings, which are used to derive the estimated error. The newly updated estimated error is compared with the previous estimations. If the variation is within a certain threshold, we consider the system stabilized and the current results to be the best estimation and final output. If the system is not stabilized yet, the virtual evidence, which describes the error distributions of the input data, is updated using the new estimated concentration and subsequently used by the Bayesian network to generate the estimated ground truth readings for the next round of optimization. The loop continues after a certain number of runs or until the system converges.

\section{Basic Bayesian belief network}

In this section, we first introduce the basic Bayesian belief network. Then we discuss how to implement it in real-world applications.

\subsection{Bayesian network introduction}

Bayesian networks are widely used to detect and recover abnormal data points for sensor networks. The Bayesian network is built based on Bayes' theorem and capable of exploiting the interdependent or causal relationships of correlated sensors readings. The types of the sensors involved can be different, which makes it appropriate for our application. A Bayesian network is a directed graph consisting of nodes and arcs (Kay, 1998).

Figure 2 shows an example Bayesian belief network for a simple sensor network. In this application, there are three different types of sensors, which can measure temperature $(T)$, carbon monoxide $(\mathrm{CO})$, and nitrogen dioxide $\left(\mathrm{NO}_{2}\right)$. 


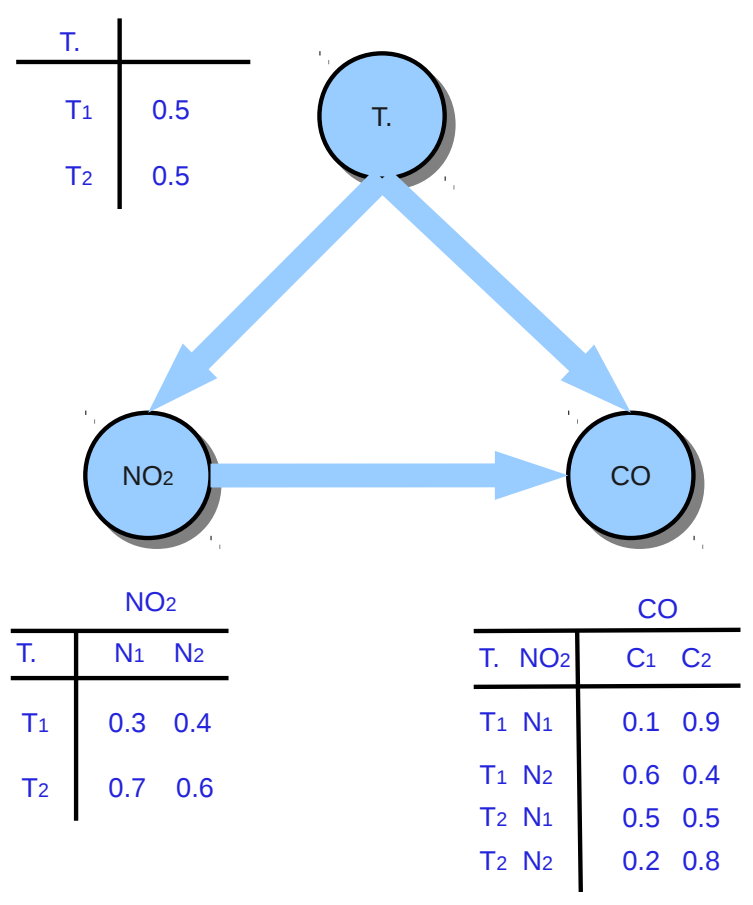

Figure 2. An example of a Bayesian belief network.

Each sensor's readings can be discretized into $N$ values, with each discrete value denoted as $T_{n}, \mathrm{C}_{n}$, and $\mathrm{NO}_{n}$, respectively. Without loss of generality, we assume two distinct discrete values for each sensor type. All the metal oxide sensors are correlated because of cross sensitivity. The readings of metal oxide sensors are strongly affected by the temperature. We found no significant impact from relative humidity.

As shown in the figure, the Bayesian network describing this sensor network contains three nodes, with each representing one type of sensor. There are two arcs connecting the temperature sensor with the metal oxide sensors and one arc connecting the two metal oxide sensors. To calculate the probability inference of each variable given the input of other variables as evidence, each node is associated with a table, which is called conditional probability table (CPT). CPT describes the conditional dependence between any node with its parents. For the root node with no parents, CPT describes the distribution of the variable itself. CPT can be derived by training the network using historic data.

\subsection{Bayesian network for real-world applications}

Without loss of generality, we assume that there are four types of equipped sensors: temperature, $\mathrm{NO}_{2}, \mathrm{CO}$, and ozone $\left(\mathrm{O}_{3}\right)$. Their readings are all correlated. The Bayesian network graph for this application is shown in Fig. 3. In the graph, there are two types of nodes. The first type, which contains $T, \mathrm{CO}(s), \mathrm{NO}_{2}(s)$, and $\mathrm{O}_{3}(s)$, represents the readings of the sensors. The second type, which contains $\mathrm{CO}(t), \mathrm{NO}_{2}(t)$, and

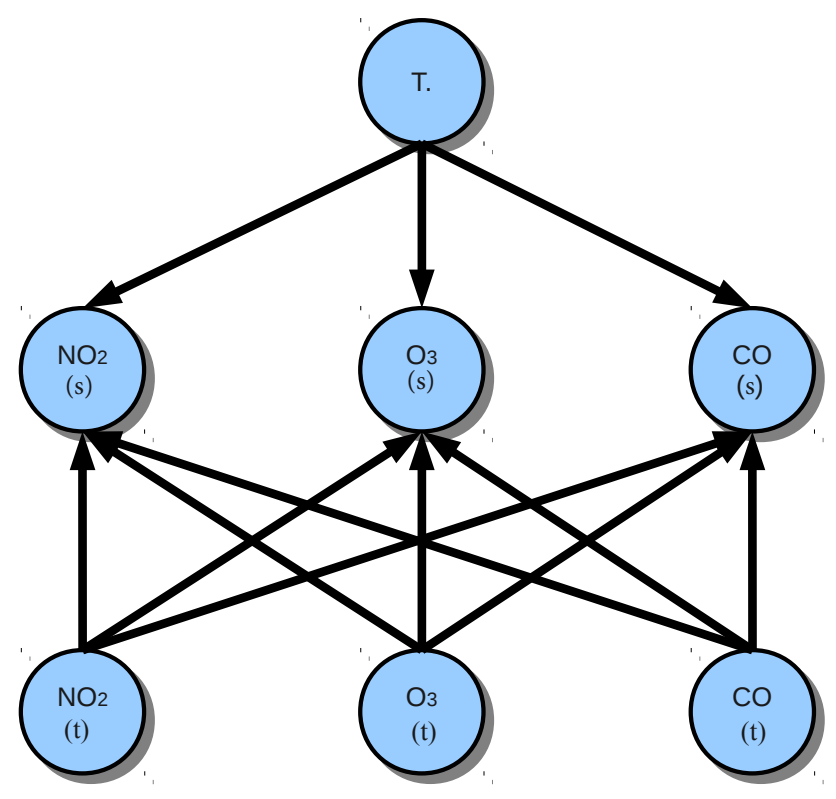

Figure 3. The basic Bayesian network structure for our application. (Symbols with $(s)$ are referred to the sensor readings; symbols with $(t)$ are referred to the ground truth concentrations.)

$\mathrm{O}_{3}(t)$, represents the actual concentration (ground truth) of the corresponding pollutants in the environment.

In the figure, there are arrows connecting the temperature sensor to all the three types of metal oxide sensors since the temperature influences the measurement results of all the three metal oxide sensors. The metal oxide sensors are assumed to be independent from each other, and the same is true for the ground truth concentration nodes. However, because of cross sensitivity, each ground truth reading can have significant impact on the readings of three metal oxide sensors simultaneously. Thus, there are three arcs connecting the ground truth concentrations to all the three sensors. When the ground truth is not available, the probability inference of the three ground truth nodes can be calculated using the input of the four actual sensors. The value with the highest probability is considered as the estimated ground truth, as shown in the following equation.

$G(i)=\max _{n \in N(i)}\left(P_{n}(i)\right)$,

where $G$ is the ground truth reading for sensor $i, N$ is the number of possible values after discretization, and $P$ is the probability.

\section{Bayesian network with sensor recalibration}

In this section, we first talk about the problems of the basic Bayesian network for real-world applications in which sensors may drift. Then we introduce virtual evidence to address 
the drift problem and the sensor recalibration technique to improve the performance of the Bayesian network. Finally, we present the combined recursive system and describe the details and algorithm to implement it.

\subsection{Problems for basic Bayesian network}

Bayesian network can clean the corrupted data and detect abnormal readings by leveraging the interdependency of correlated sensors. For the random noises, it is quite efficient and sufficient. However, in our applications, sensors frequently drift. It has been shown, both in existing literature (Xiang et al., 2012; Romain and Nicolas, 2010) and by our own measurement data presented in Sect. 7.1.3, that sensor drift is a very common and severe problem in real-world applications for those metal oxide sensors. Sensor readings become useless with the bias observed in our deployment. Thus, the problem of sensor drift and the error caused by drift must be addressed.

The basic Bayesian network is based on the assumption of unbiased measurements. Thus, it is unable to generate reasonable results when multiple sensors drift simultaneously. Note that it is quite common to have more than one drifted sensors in the system simultaneously, as shown by our deployment results in Sect. 7.1. Thus, the system described in Fig. 3 is inadequate to address the real-world problems. To apply the Bayesian network in such circumstances, we need to (1) incorporate a ranking mechanism that can quantify the sensor uncertainties into the Bayesian network and (2) design a drift compensation scheme to recalibrate the sensor function and recover the corrupted data simultaneously within the Bayesian network framework.

\subsection{Error distribution and uncertain evidence}

As the sensor drifts, its sensing sensitivity deteriorates and the uncertainty of its readings increases. A Bayesian network treats all its input equally, which is problematic considering sensor drifts. For example, if a CO sensor is recently calibrated while an $\mathrm{O}_{3}$ sensor has not been calibrated for a long time, we should clearly give the $\mathrm{CO}$ sensor more confidences. In other words, within a Bayesian network framework, we must have an evaluation mechanism which can rank and quantify the trustworthiness of each particular sensor.

To address this problem, we use error distributions to represent the sensitivity and trustworthiness of the sensors. An example of error distributions is shown in Table 1. In the example, we assume that the sensor has reported an environment concentration of $1.5 \mathrm{ppm}$. The actual ground truth ranges from 0 to $3 \mathrm{ppm}$ and is divided into three discrete categories. We assume that in the environment the probability for the ground truth to be in any of these three categories is equal. As shown in Table 1, if the sensor is accurate, then the probability that the actual ground truth is within the range of
Table 1. An example error distribution with reported reading of $1.5 \mathrm{ppm}$.

\begin{tabular}{l|ccc}
\hline & \multicolumn{3}{|c}{ Ground truth prob. (\%) } \\
& $0 \sim 1 \mathrm{ppm}$ & $1 \sim 2 \mathrm{ppm}$ & $2 \sim 3 \mathrm{ppm}$ \\
\hline Accurate & 0 & 100 & 0 \\
Drifted & 30 & 70 & 0 \\
Breakdown & 33 & 33 & 33 \\
\hline
\end{tabular}
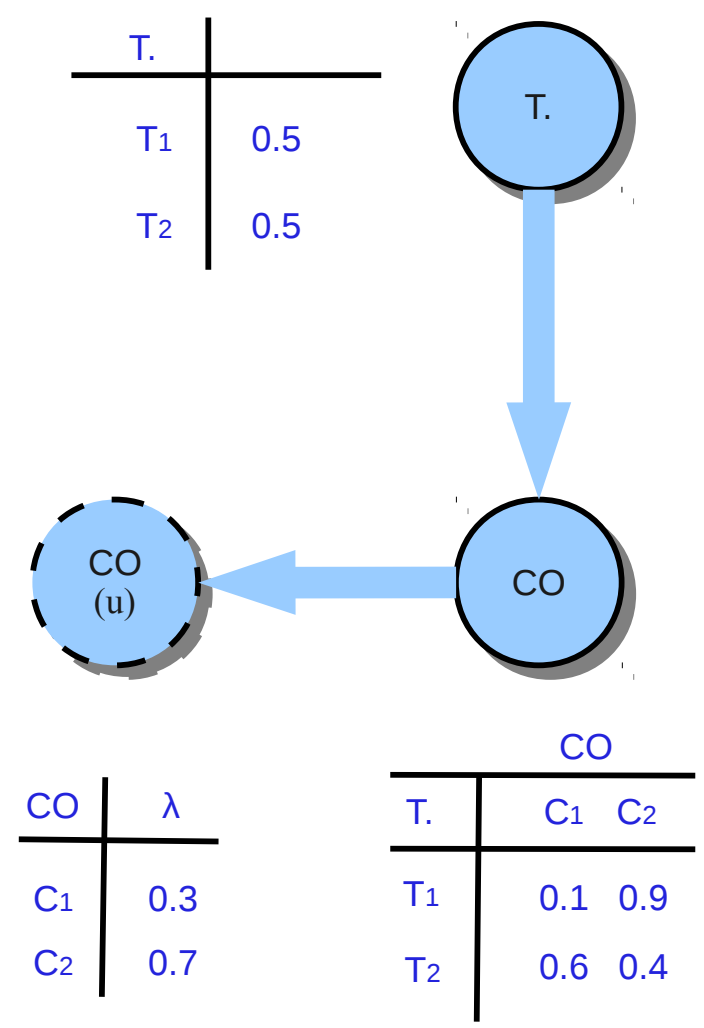

Figure 4. An example of virtual node. (Symbol with $(u)$ is referred to the virtual node.)

1 to $2 \mathrm{ppm}$ given a reported reading of $1.5 \mathrm{ppm}$ is $100 \%$. If the sensor is drifted, the sensor becomes less accurate and the possible value of the ground truth spreads wider. If the sensor has a breakdown, it loses most of its sensitivity and the ground truth is no longer correlated to the sensor readings.

In that way, we have transformed the determined sensor readings into distributions which inherently represent the trustworthiness of the sensors. Such input to the Bayesian network is called virtual evidence. Note that virtual evidence cannot be applied to the Bayesian network directly. The Bayesian network must be modified to incorporate such uncertain evidence. 
Table 2. The statistics of the original and drifted sensor readings.

\begin{tabular}{l|ccc|ccc}
\hline \multirow{2}{*}{ Errors } & $\begin{array}{c}\mathrm{CO} \\
\text { Undrifted } \\
\mathrm{NO}_{2}\end{array}$ & $\begin{array}{c}\mathrm{O}_{3} \\
(\mathrm{ppb})\end{array}$ & $\begin{array}{c}\mathrm{CO} \\
(\mathrm{ppm})\end{array}$ & $\begin{array}{c}\text { Drifted } \\
\mathrm{NO}_{2} \\
(\mathrm{ppm})\end{array}$ & $\begin{array}{c}\mathrm{O}_{3} \\
(\mathrm{ppm})\end{array}$ \\
\hline Average & 0.31 & 16.13 & 0.04 & 10.72 & 112.45 & 0.20 \\
Maximum & 8.92 & 76.11 & 0.32 & 21.94 & 171.4 & 1.85 \\
Standard deviation & 0.52 & 11.19 & 0.07 & 0.93 & 12.50 & 0.28 \\
\hline Correlation & \multicolumn{7}{c}{$93 \%$} \\
\hline
\end{tabular}

\subsection{Bayesian network with virtual evidence}

For the basic Bayesian network, the inputs can only be determined values. To incorporate the virtual evidence, some constraints must be honored, which is called Jeffrey's rule (Jeffrey, 1990). The concept of Jeffrey's rule is described as follows.

Suppose the universe of all the events is denoted as $U$. We have a set of mutually exclusive events $\gamma_{1}, \ldots, \gamma_{n}$, which is a subset of $U$, and $P$ is the probability distribution of those events. After applying the virtual evidence, the beliefs for events $\gamma_{1}, \ldots, \gamma_{n}$ change and the updated distribution is denoted as $P^{\prime}$. $P^{\prime}$ should satisfy the following equation.

$P\left(\alpha \mid \gamma_{i}\right)=P^{\prime}\left(\alpha \mid \gamma_{i}\right), \forall i=1, \ldots, n$,

where $\alpha$ is any event in the universe. In other words, after the virtual evidence is accepted, the posterior probability of $\alpha$ can be changed, but the conditional probability for $\alpha \in U$ regarding to the events $\gamma_{1}, \ldots, \gamma_{n}$ must remain the same.

To treat the virtual evidence as determined value while honoring the Jeffrey's rule, the Bayesian network should be modified by adding a virtual node to the drifted sensor nodes (Chan and Darwiche, 2005). Figure 4 shows an example Bayesian network with virtual nodes. In the figure, the pollutant followed by $V$ represent a virtual node in the Bayesian network. The number in Table 2 is the conditional probability. $\lambda$ represents the probability distribution of the input evidence. There are two sensor nodes: temperature and $\mathrm{CO}$. The temperature sensor is assumed to be accurate and with little drift, while the $\mathrm{CO}$ sensor can drift. The $\mathrm{CO}$ sensor node is associated with a virtual node, denoted as $\mathrm{CO}(u)$. The virtual node also has its own conditional probability table. The CPT of the virtual node should be calculated using the error distribution of the actual sensor node so that the beliefs of the whole Bayesian network comply with Jeffrey's rule. The detailed methods and equations to calculate its probability table can be found in existing literature (Peng et al., 2010; Chan and Darwiche, 2005). Note that the virtual node is only dependent on the corresponding sensor node and independent of all the other nodes in the network.

Figure 5 shows the Bayesian network structure of our application after incorporating the virtual evidence. Since the temperature sensor and the hypothetical ground truth con-

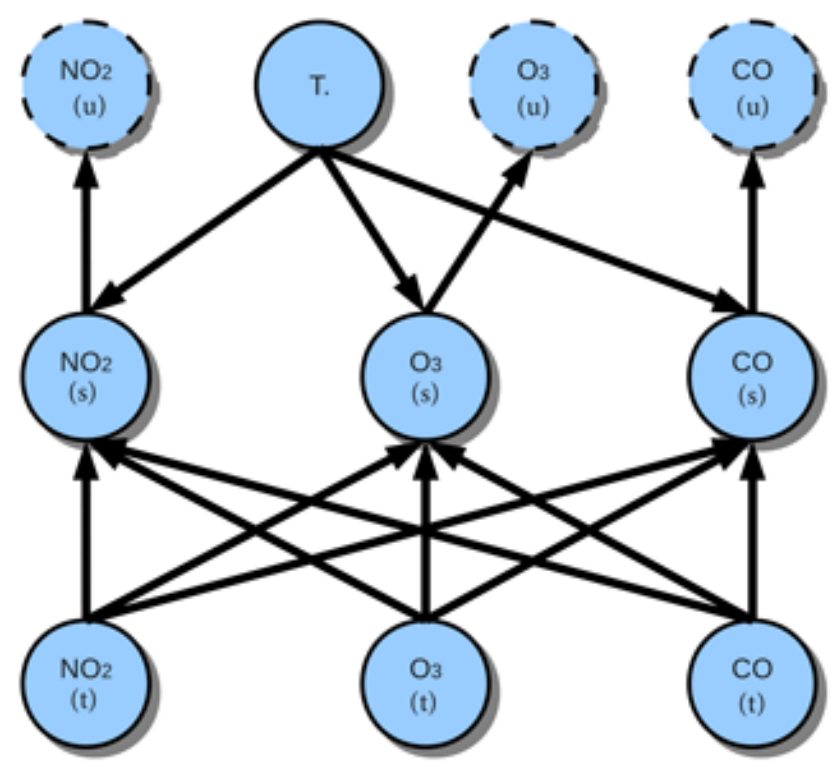

Figure 5. The Bayesian network with virtual nodes. (Symbol definition in Sect. 5.2.)

centration sensors are assumed to be accurate, they are not associated with any virtual nodes. Each metal oxide sensor, which is prone to drift, is associated with a virtual node. The contents in the CPT of the virtual nodes can be calculated using the error distributions of the actual nodes, which can be derived with the information of the (estimated) ground truth readings and the sensor readings.

\subsection{Sensor function recalibration}

The transformation function to translate the analog input signal into pollutant concentration is called a sensor calibration function, or sensor function. The abnormal readings caused by environmental noises do not reflect a change of the sensor function. However, when sensors are drifted, the sensor functions change, which can cause a systematic increase of abnormal readings.

In this work, we apply a piece-wise linear function as the sensor function, which is shown in the following equation.

$C=p_{1}+p_{2} \times V+p_{3} \times T$, 
where $C$ is the pollutant concentration, $p_{i}$ are the fitting parameters, $V$ is the voltage, and $T$ is the temperature. The temperature information is reported by the on-board sensors. Note that for our experimented sensors, the impact of humidity is much less significant than temperature. Thus, we do not include humidity in our setup. If parameters in other applications, such as humidity and pressure, do have significant impact, they can be easily incorporated to our Bayesian framework. The parameters in the equation are derived using linear regression with the training data. Since accurate sensors providing ground truth readings are usually not available, we use the estimated ground truth concentration returned by the Bayesian network instead. Note that as the sensitivity of the sensors reduces, the performance of this recalibration scheme deteriorates. When a sensor breaks down and loses most of its sensitivity, the sensor can no longer be recalibrated.

\section{Experimental results}

In this section, we first describe a real-world co-location deployment of nine mobile sensor nodes and the analysis results for the deployment data. We then evaluate our system using the real-world data.

\subsection{Mobile sensor network deployment and analysis}

\subsubsection{The mobile sensing device}

To investigate the effect of sensor drift in real-world applications and collect data to evaluate our data cleaning technique, we deployed a sensor network in Denver, Colorado. During the experiment, we deployed nine M-Pods (Jiang et al., 2011), which are shown in Fig. 6. The M-Pod is a custombuilt mobile sensing device supporting embedded sensing, computation, and wireless communication. It supports detection of various air pollutants, including $\mathrm{NO}_{2}, \mathrm{CO}, \mathrm{CO}_{2}, \mathrm{O}_{3}$, and volatile organic compounds. It can also measure temperature, humidity, and light. The latest revision of the M-Pod is compact $(5 \times 6.5 \mathrm{~cm})$ and energy efficient, with a battery life of greater than $16 \mathrm{~h}$. The whole device, including a Li-ion battery with a capacity of $6000 \mathrm{~mA}-\mathrm{h}$, is enclosed by a lowcost off-the-shelf case that can be carried using an armband or attached to a backpack. A [3.3] V DC fan is used to control airflow. A rectangular filter is installed around sensor to increase sensing accuracy and prolong sensor life. Most of the power-hungry on-board sensors are power gated and can be controlled by commands from smartphones. Data are temporally stored in a 1 megabyte non-volatile EEPROM. The total cost of the on-board components and sensors is less than USD 150 and can be reduced further when produced in large quantities.

To receive, store, and present the data gathered by our MPod device, we have developed on-board firmware, smartphone applications, data servers, and Web interfaces. The firmware defines protocols of sensing, storing, and sending the environmental data. The smartphone application communicates with the M-Pod via its Bluetooth interface. It can issue commands to and receive data from the M-pod. The data are transmitted to the online data server and stored in the databases. A Web based user interface allows users to access and analyze air quality data.

\subsubsection{The real-world deployment}

The nine M-Pods were used continuously from March to May 2013. The sensors were not changed throughout this period. For the majority of the time, the M-Pods were worn by users as part of an exposure assessment study. During three multi-day calibration periods in March, April, and May, the M-Pods were placed at a reference air quality monitoring site. The M-Pods were powered continuously on the roof of the monitoring building, in a ventilated enclosure near the air inlets for the reference monitors. The reference site, as shown in Fig. 6, monitors $\mathrm{CO}, \mathrm{NO}_{2}$, and $\mathrm{O}_{3}$. It is located in downtown Denver, Colorado, and operated by the Colorado Department of Public Health and Environment (CDPHE). The highly accurate and regularly maintained air pollutant monitoring equipment in the station is used to provide the ground truth readings.

By co-locating the M-Pods with the reference monitors, we are able to derive both the sensor analog readings and ground truth, which can be used to determine the sensor calibration functions. The forms of the sensor calibration functions vary depending on sensor type. In this work, we use a piece-wise linear function. It is quite accurate according to lab and field measurements and requires much fewer resources to compute compared to other, more complicated, forms of sensor functions. The calibrations are performed using the field data. Thus, it does not require specialized equipment and can cover a wider range of environmental parameter space than lab calibrations. Before the fitting of the sensor function, data filtering was performed to remove noise from the sensor readings. Minute medians were first calculated from the $6 \mathrm{~s}$ raw data. Then, we applied a filter based on difference in consecutive differences in the medians. There were two thresholds for the filter: an absolute threshold that was deemed unrealistic based on lab experiments and 2 times the standard deviation of the differences. By performing calibrations periodically with the same sets of sensors, we were able to assess the change in baseline readings and sensitivity over time. The calibration functions derived by fitting to the data of the first calibration period, which is considered as the undrifted baseline, are applied to the entire data set.

\subsubsection{Data analysis}

We examine and compare the readings of the $\mathrm{CO}, \mathrm{NO}_{2}$, and $\mathrm{O}_{3}$ sensors. An example of the measured data and the corresponding ground truth readings is presented in Fig. 7. The $X$ axis in the figure shows the time line of the deployment 


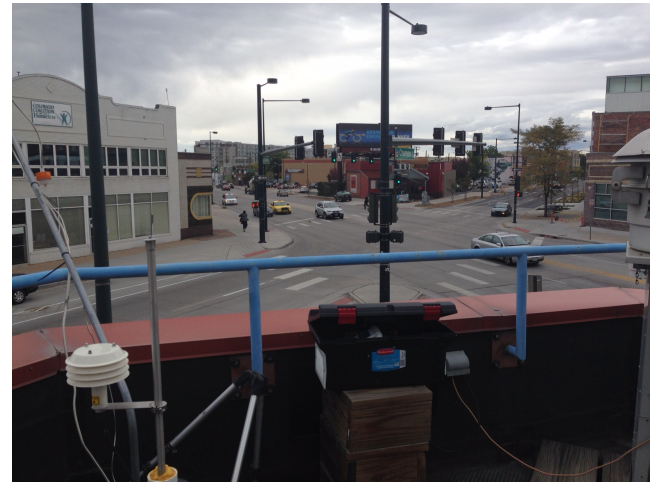

(a)

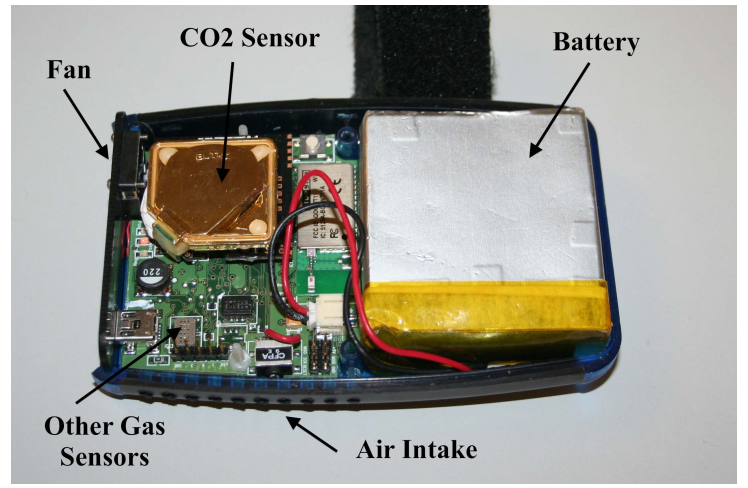

(b)

Figure 6. (a) The Denver air quality monitoring station; (b) the M-Pod sensing platform.

in the unit of days, while the $Y$ axis shows the concentration of the pollutant in parts per million. Two sets of data are presented: ground truth data and M-pod measured data. The total duration of the deployment is about 2 months. In the figure, there are three separate time periods, with each lasting for about 1 week. During that time period, the M-Pods are located in the station and calibrating. For the rest of the time, the M-Pods are carried by individual users and the ground truth readings of their exposed environments are unknown. Thus, the readings from those time periods are not included.

The resultant data show that the drift rates for different types of sensors vary. For the example in the figure, the $\mathrm{NO}_{2}$ sensor experiences large drift. After 2 months, its drift error is increased more than 3 times. The CO sensor also suffers significant drift, though less compared to the $\mathrm{NO}_{2}$ sensor with about $50 \%$ increase of error. However, for the $\mathrm{O}_{3}$ sensor, no significant drift is observed. The example shows that significant drift can occur within just a couple of months, rendering the corresponding sensor almost useless if not carefully recalibrated. It demonstrated that drift is a real and severe challenge for those cheap sensors to be useful in realworld applications. Moreover, since the exposed environment and the properties of the sensors vary, different sensors usually exhibit different drift rates, making it impossible to recalibrate the sensors using a predetermined model.

Among the nine M-Pods deployed, we chose six of them during our analysis and evaluations. For the rest three, one of them did not return enough data due to transmission problem, and two of them have sensors completely dead within the 2month deployment period. Table 2 shows the statistics of the sensing errors from the remaining six M-Pods. The error in the table are defined as the absolute variation between the sensor reading and the ground truth. We compare the drifted and undrifted data. The undrifted data are taken from the first time period as shown in Fig. 7. The drifted data are taken from the third time period. The first three rows show the average, maximum, and standard deviation of the error distri- butions. Significant drift can be observed for all the types of sensors. It should be noted that for some pollutants, such as $\mathrm{NO}_{2}$ and $\mathrm{CO}$, their mean values change more significantly than the standard deviation, which implies a close to linear shift. For each pair of sensors, e.g., $\mathrm{NO}_{2}$ and $\mathrm{O}_{3}$, their correlation coefficient is calculated. Among all the possible pairs, $93 \%$ of them show a strong correlation, indicating that the Bayesian network might be an appropriate solution.

In conclusion, our deployment data show that sensor drift and consequently the noise problem are very realistic and important for the metal oxide sensors. If not properly addressed, most of those sensors will be useless within just a couple of months. The drift rates are dependent on the environment and sensor properties and, hence, vary for different sensors. Thus, it is not feasible to use predetermined correction methods; sensor calibration problem must be addressed using the field data. Moreover, different types of sensors show strong correlations, permitting noise reduction and sensor calibration.

\subsection{Data recovery and sensor calibration results}

\subsubsection{Experiment setup}

The outlier cleaning and sensor recalibration functions are written using Matlab, with the help of an external Bayesian network toolbox called bnt (Bayes toolbox, 2007). We use the data returned from six out of a total of nine sensors deployed, excluding the failed sensors and sensors with insufficient data. The failed sensors are not used since their readings are no longer correlated with each other and recalibration cannot help improve the results. In other words, our technique does not have effect on them and they should be simply replaced. The failed sensor can be detected using both our technique and the Bayesian network method. The threshold to determine the outliers is taken equal to the standard deviation of the ground truth readings. 


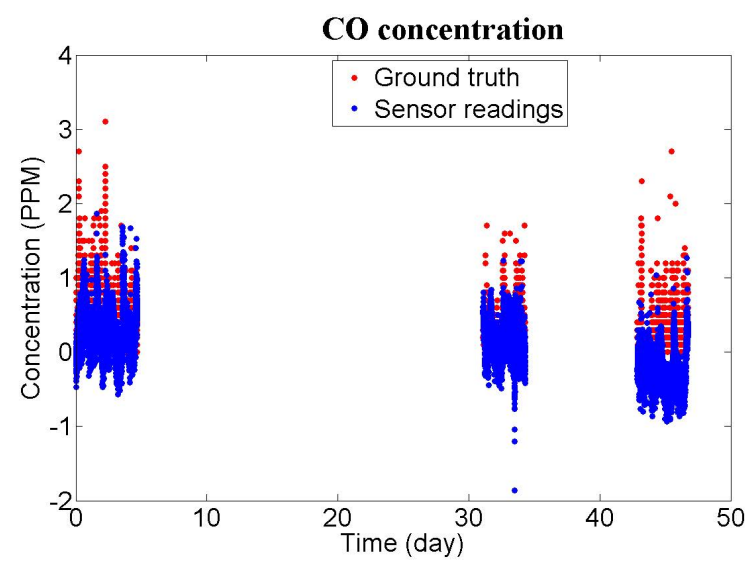

(a)

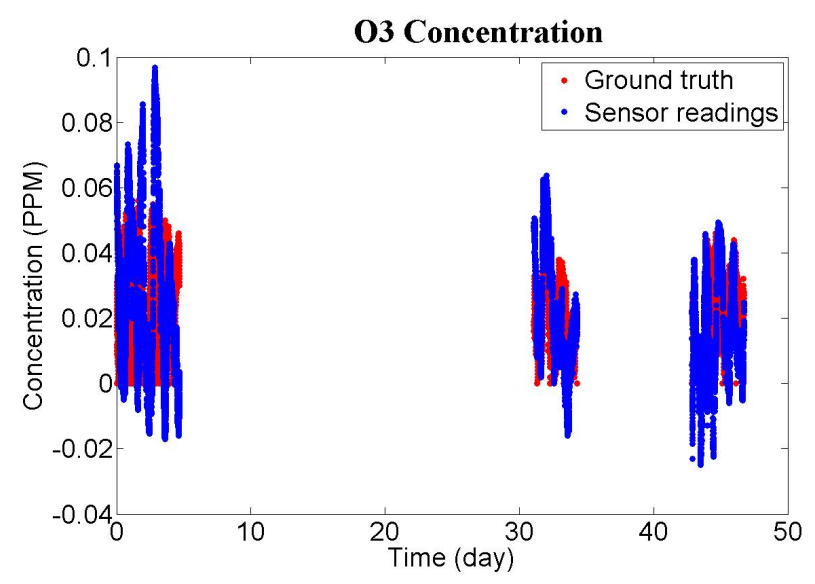

(b)

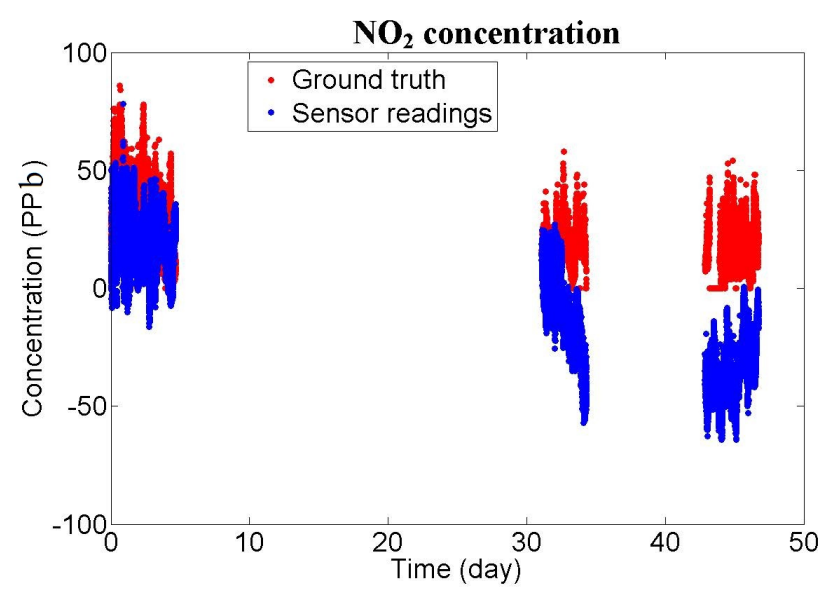

(c)

Figure 7. (a) Drift measurement of CO; (b) drift measurement of $\mathrm{O}_{3} ;$; c) drift measurement of $\mathrm{NO}_{2}$.
The CPT of the Bayesian network is derived from training. The training set is generated using the co-location data from the undrifted (the first) time period. This approach is more appropriate since it requires much less effort to cover a reasonable number of states than lab environment and can provide us a more realistic prior distributions for temperature. The training data set is filtered so that it contains only normal data. After the Bayesian network is trained, the contents in the CPT remain unchanged until the sensor is close to a reference station and have access to the ground truth readings again. For the parameter states that are not encountered during the training phase, we replace their contents with the encountered state of the closest distance, calculated using the Euclidean distance between those two states.

To evaluate our outlier recovery and sensor recalibration technique, we compare the following three approaches.

1. Uncompensated: this approach interprets the reported analog data using the predetermined sensor function from lab measurement and without any compensation scheme.

2. Bayesian network: this approach implements a Bayesian belief network based technique proposed by Janakiram et al. (2006). It is the most relevant and closely related work to the best of our knowledge.

3. Bayesian network with virtual evidence: this is our proposed technique. It improved upon the Bayesian network approach by incorporating the virtual evidence and sensor recalibration.

We evaluate all the three approaches using the same set of testing data derived from our real-world deployment.

\subsubsection{Drifted sensor recovery evaluation}

Many existing outlier detection approaches, such as distance-based techniques (Papadimitriou et al., 2003; Subramaniam et al., 2006) or classification-based techniques (Rajasegarar et al., 2007), cannot estimate the ground truth data and provide recalibration opportunities for the drifted sensors. Thus, we do not include them in the comparison. Figure 8 shows the performance of various relevant data cleaning and recovery techniques. Since our technique focuses on the sensor drift and recalibration problem, the experiment is performed on the third time period of the data set, which represents the drifted sensors. The $Y$ axis of the bar graph shows the average errors, which are normalized to our recursive technique. The red numbers above the bar show the actual average error value for the uncompensated method. Compared with the uncompensated approach, in which the sensor outliers are not compensated and sensor calibration functions are not recalibrated, our technique can incur only 


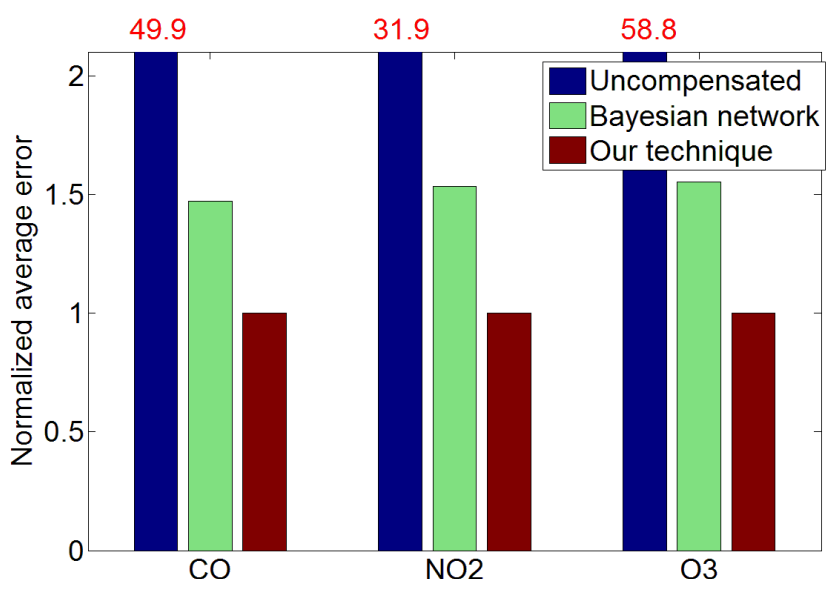

Figure 8. The data recovery results of various techniques for the drifted data.

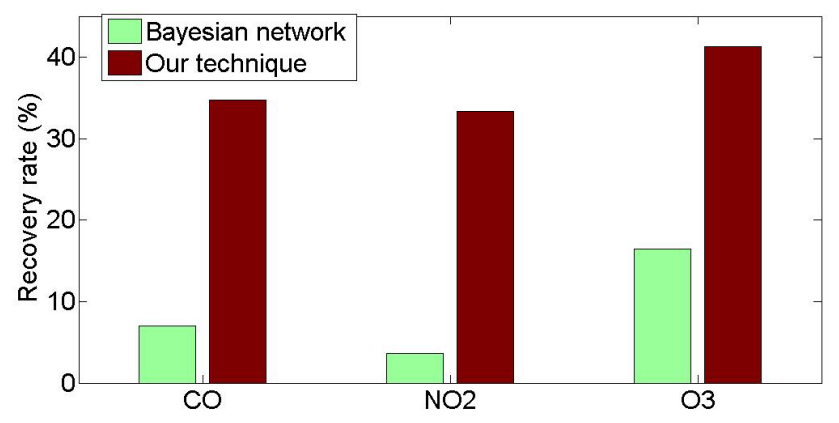

Figure 9. The percentage of successfully cleaned data.

about $2.13 \%$ error on average. Moreover, compared with the Bayesian network approach, which is the closest existing technique, our technique is capable of reducing errors by $32.0,34.7$, and $35.5 \%$ for $\mathrm{CO}, \mathrm{NO}_{2}$, and $\mathrm{O}_{3}$, respectively. In our setup, the experiment results show that our technique can reduce error by $34 \%$ on average.

After the estimated ground truth values are derived, we consider it the ground truth concentration. However, since the ground truth concentration estimation is imperfect, the classification of sensor readings according to this estimate ground truth concentrations can be wrong. Hereby we define data recovery rate as the percentage of corrected label data points after the data recovery scheme. Figure 9 shows the comparison results of various techniques in terms of data recovery rate. The rate is obtained by comparing the estimated readings against the ground truth. For our technique, the data recovery rates are $34.7,33.3$, and $41.3 \%$ for $\mathrm{CO}$, $\mathrm{NO}_{2}$, and $\mathrm{O}_{3}$, respectively. Compared with the Bayesian network approach, our technique successfully recovers 4 times more data.

\section{Conclusions}

In this work, we have presented a Bayesian belief network based system to detect and recover outliers in the presence of sensor drift. This work is to address the data noise and sensor drift problems in atmospheric research by exploring the correlation of different types of sensors. In our analysis of real-world data, low-cost air quality sensors usually incur significant drift within a few months. Thus, to ensure the accuracy of the atmosphere researches utilizing those sensors, we developed a data treatment technique that can significantly reduce the sensor noise and recalibrate the drifted sensor online.

Our technique can significantly improve sensor accuracy given the cross-correlation of sensors for different gasses. Thus, it is most suitable for applications involving sensor clusters, such as mobile environmental sensing, health monitoring, and hazard gas alert. However, for the more traditional monitoring methods, which rely upon accurate and singular sensors, the applications of our technique are more limited.

Nonetheless, there are many areas for improvements in the future research. This technique, along with other Bayesian network based techniques, requires extensive training prior deployment. However, with limited training data, offline training can introduce inaccuracies with untrained events. Thus, it is important to develop online Bayesian network based training techniques alongside the calibration technique. Moreover, the response time of different sensors may differ with each other. Thus, the technique should be adapted to address sensors with variable response time. Bayesian network based techniques can also be used for sensor network malfunctioning analysis and diagnosis, which is another important application area.

Edited by: P. Stammes

\section{References}

Arshak, K., Moore, E., Lyons, G. M., Harris, J., and Clifford, S.: A review of gas sensors employed in electronic nose applications, Sensor Rev., 24, 181-198, 2004.

Bayes toolbox: Bayes Net Toolbox for Matlab, https://code.google. com/p/bnt/, last access date: 19 October 2007.

Bettencourt, L. M., Hagberg, A., and Larkey, L.: Separating the Wheat from the Chaff: Practical Anomaly Detection Schemes in Ecological Applications of Distributed Sensor Networks, Lect. Notes Comput. Sc., 4549, 223-239, 2007.

Bychkovskiy, V., Megerian, S., Estrin, D., and Potkonjak, M.: A collaborative approach to in-place sensor calibration, Lect. Notes Comput. Sc., 2634, 301-316, 2003.

Chan, H. and Darwiche, A.: On the revision of probabilistic beliefs using uncertain evidence, Artif. Intell., 163, 67-90, 2005.

Chandola, V., Banerjee, A., and Kumar, V.: Anomaly detection: A survey, ACM Comput. Surv., 41, 15:1-15:58, 2009.

Di Lecce, V. and Calabrese, M.: Discriminating gaseous emission patterns in low-cost sensor setups, IEEE International Con- 
ference on Computational Intelligence for Measurement Systems and Applications (CIMSA), 19-21 September 2011, Ottawa, Canada, 1-6, 2011.

Elnahrawy, E. and Nath, B.: Cleaning and querying noisy sensors, WSNA '03 Proceedings of the 2nd ACM international conference on Wireless sensor networks and applications, 19 September 2003, San Diego, CA, USA, 78-87, 2003.

Haugen, J.-E., Tomic, O., and Kvaal, K.: A calibration method for handling the temporal drift of solid state gas-sensors, Anal. Chim. Acta, 407, 23-39, 2000.

Janakiram, D., Adi Mallikarjuna Reddy, V., and Phani Kumar, A.: Outlier Detection in Wireless Sensor Networks using Bayesian Belief Networks, First International Conference on Communication System Software and Middleware, 2006, Comsware 2006, New Delhi, India, 1-6, 2006.

Jeffrey, R. C.: The logic of decision, University of Chicago Press, Chicago, USA, 1990.

Jiang, Y., Li, K., Tian, L., Piedrahita, R., Xiang, Y., Mansata, O., Lv, Q., Dick, R. P., Hannigan, M., and Shang, L.: MAQS: A personalized mobile sensing system for indoor air quality monitoring, UbiComp '11 Proceedings of the 13th international conference on Ubiquitous computing, 17-21 September 2011, Beijing, China, 271-280, 2011.

Kay, S. M.: Fundamentals of Statistical signal processing, Volume 2: Detection theory, Prentice Hall PTR, Upper Saddle River, New Jersey, USA, 1998.

Kumar, D., Rajasegarar, S., and Palaniswami, M.: Automatic Sensor Drift Detection and Correction Using Spatial Kriging and Kalman Filtering, in: Proc. Int. Conf. Distributed Computing in Sensor Systems, pp. 183-190, 2013.

Miluzzo, E., Lane, N., Campbell, A., and Olfati-Saber, R.: CaliBree: A Self-calibration System for Mobile Sensor Networks, Lect. Notes Comput. Sc., 5067, 314-331, 2008.

Papadimitriou, S., Kitagawa, H., Gibbons, P., and Faloutsos, C.: LOCI: fast outlier detection using the local correlation integral, IEEE 19th International Conference on Data Engineering (ICDE’03), 5-8 March 2003, Bangalore, India, 315-326, 2003.

Peng, Y., Zhang, S., and Pan, R.: Bayesian network reasoning with uncertain evidences, J. Uncertainty, Fuzziness and KnowledgeBased Systems, 18, 539-564, 2010.

Piedrahita, R., Xiang, Y., Masson, N., Ortega, J., Collier, A., Jiang, Y., Li, K., Dick, R. P., Lv, Q., Hannigan, M., and Shang, L.: The next generation of low-cost personal air quality sensors for quantitative exposure monitoring, Atmos. Meas. Tech., 7, 3325-3336, doi:10.5194/amt-7-3325-2014, 2014.
Rajasegarar, S., Leckie, C., Palaniswami, M., and Bezdek, J.: Quarter Sphere Based Distributed Anomaly Detection in Wireless Sensor Networks, IEEE International Conference on Communications, ICC '07, 24-28 June 2007, Glasgow, UK, 3864-3869, 2007.

Romain, A. and Nicolas, J.: Long term stability of metal oxidebased gas sensors for e-nose environmental applications: An overview, Sensor. Actuat. B-Chem., 146, 502-506, 2010.

Subramaniam, S., Palpanas, T., Papadopoulos, D., Kalogeraki, V., and Gunopulos, D.: Online outlier detection in sensor data using non-parametric models, VLDB '06 Proceedings of the 32nd international conference on Very large data bases, 12-15 September 2006, Seoul, Korea, 187-198, 2006.

Tans, P. and Thoning, K.: How we measured background $\mathrm{CO}_{2}$ levels on Mauna Loa., available at: http://www.esrl.noaa.gov/ gmd/ccgg/about/co2_measurements.html, last access: September 2008 .

Willett, W., Aoki, P., Kumar, N., Subramanian, S., and Woodruff, A.: Common Sense Community: scaffolding Mobile Sensing and Analysis for Novice Users, Lect. Notes Comput. Sc., 6030, 301318, 2010.

Xiang, Y.: Mobile Sensor Network Design and Optimization for Air Quality Monitoring, Ph.D. thesis, The University of Michigan, Ann Arbor, MI, USA, 2014.

Xiang, Y., Bai, L. S., Piedrahita, R., Dick, R. P., Lv, Q., Hannigan, M. P., and Shang, L.: Collaborative calibration and sensor placement for mobile sensor networks, ACM/IEEE 11th International Conference on Information Processing in Sensor Networks (IPSN), 16-20 April 2012, Beijing, China, 73-84, 2012.

Xiang, Y., Piedrahita, R., Dick, R., Hannigan, M., Lv, Q., and Shang, L.: A Hybrid Sensor System for Indoor Air Quality Monitoring, IEEE International Conference on Distributed Computing in Sensor Systems (DCOSS), 20-23 May 2013, Cambridge, MA, USA, 96-104, 2013.

Zampolli, S., Elmi, I., Ahmed, F., Passini, M., Cardinali, G., Nicoletti, S., and Dori, L.: An electronic nose based on solid state sensor arrays for low-cost indoor air quality monitoring applications, Sensor. Actuat. B-Chem., 101, 39-46, 2004.

Zhang, Y., Meratnia, N., and Havinga, P.: Outlier Detection Techniques for Wireless Sensor Networks: A Survey, IEEE Communications Surveys Tutorials, 12, 159-170, 2010. 